\title{
Der hauptamtliche Präsident des Europäischen Rates: ,Herr' oder ,Diener' im Haus Europa?
}

\author{
Wolfgang Wessels und Thomas Traguth*
}

Der Europäische Rat kann seit seiner Gründung im Jahre 1974 als Pionier und Schlüssel zur Evolution des politischen Systems der Europäischen Union gelten. Er besitzt de facto Entscheidungsmacht bei allen maßgeblichen Schritten und Meilensteinen der Entwicklung der Europäischen Union. ${ }^{1}$ Die neuen Artikel des Vertrages von Lissabon eröffnen eine frische Perspektive auf das geschriebene Regelwerk und die gelebte Alltagspraxis des Europäischen Rates.

In seiner Rolle als Politikgestalter spiegelt der Europäische Rat in über 110 Sitzungen seit $1975^{2}$ die zunehmende Bandbreite innen- wie außenpolitischer Themenfelder auf seiner Tagesordnung wider. Er legt Leitlinien zu den zahlreichen Politikfeldern fest, so zum Beispiel mit den Programmen von Tampere ${ }^{3}$ und Stockholm ${ }^{4}$ für den Raum der Freiheit, der Sicherheit und des Rechts wie auch mit der Lissabon-Strategie ${ }^{5}$ und der Europa 2020-Strategie ${ }^{6}$ für den Bereich der Beschäftigungs- und Sozialpolitik und das auswärtige Handeln der Union. Als Systemgestalter und konstitutioneller Architekt der Union beschließt er langfristig orientierte transformative Schritte, Regierungskonferenzen zur Vertragsreform, schließt faktisch Vereinbarungen zu den EU-Eigenmitteln sowie den Haushaltsprioritäten im mehrjährigen Finanzrahmen und ist Initiator und Letztinstanz in Fragen der Erweiterung. So wurde zum Beispiel unter dänischer Präsidentschaft 2002 beschlossen, die Union, basierend auf der Entscheidung von 1993, bis 2004 auf 25 Mitglieder zu erweitern. Aber auch in internen und externen Konflikten sowie außergewöhnlichen oder umstrittenen Konfliktsituationen bleibt der Europäische Rat Letztentscheidungsinstanz und verfügt über Ad-hoc-Instrumente für das interne und externe Krisenmanagement. ${ }^{7}$

Die Einführung des hauptamtlichen Präsidenten des Europäischen Rates zusammen mit der vertraglichen Aufgabenstellung des Amtes und einer eigenen Geschäftsordnung ${ }^{8}$ kann als

* Prof. Dr. rer. pol. Wolfgang Wessels, Jean Monnet Lehrstuhl, Forschungsinstitut für Politische Wissenschaft und Europäische Fragen, Universität zu Köln.

Thomas Traguth, MA (oxon) MAES (Brugge), Wissenschaftlicher Mitarbeiter, Forschungsinstitut für Politische Wissenschaft und Europäische Fragen, Universität zu Köln.

1 Vgl. Jan Werts: The European Council, London 2008; Philippe de Schoutheete: The European Council, in: John Peterson/Michael Shackleton (Hrsg.): The Institutions of the European Union, Oxford 2006, S. 37-59; Philippe de Schoutheete/Helen Wallace: The European Council, Research and Policy Group Notre Europe: Research and European Issues Nr. 19, Paris 2002; Peter Ludlow: The Leadership in an Enlarged European Union. The European Council, the Presidency and the Commission, EuroComment: Briefing Note 8/2005; Peter Ludlow: The Laeken Council, EuroComment: Briefing Note 1/2002.

2 Eigene Zählung basierend auf den Schlussfolgerungen des Europäischen Rates.

3 Rat der Europäischen Union: Schlussfolgerungen des Vorsitzes. Europäischer Rat (Tampere) 15. und 16. Oktober 1999, SN 200/99.

4 Das Stockholmer Programm - Ein offenes und sicheres Europa im Dienste und zum Schutz der Bürger, in: Amtsblatt der EU, Nr. C 115 vom 4. Mai 2010, S. 1-38.

5 Rat der Europäischen Union: Schlussfolgerungen des Vorsitzes. Europäischer Rat (Lissabon), 23. und 24. März 2000, SN 100/00.

6 Europäischer Rat: Europäischer Rat. Tagung am 17. Juni 2010. Schlussfolgerungen, Dok. EUCO 13/10.

7 Vgl. Adriaan Schout/Sophie Vanhoonacker: Evaluating Presidencies of the Council of the EU: Revisiting Nice, in: Journal of Common Market Studies 5/2006, S. 1051-1071, hier S. 1055.

8 Beschluss des Europäischen Rates vom 1. Dezember 2009 zur Festlegung seiner Geschäftsordnung (2009/882/ EU), in: Amtsblatt der EU, Nr. L 315 vom 2. Dezember 2009, S. 51-55. 
ein Hauptmoment der institutionellen Reform durch den Vertrag von Lissabon gesehen werden. Es ist mit geschärftem Blick zu beobachten, ob der Präsident die Entscheidungsverfahren und -muster sowie den Einfluss dieser Schlüsselinstitution nachhaltig prägen wird. Nicht zuletzt die Debatten des Konvents zur Zukunft Europas ${ }^{9}$ und die politische Kontroverse um die Wahl des ersten Amtsinhabers zeigen deutlich die Brisanz und Bedeutung dieser neuen Rolle für die Institution wie auch für die institutionelle Architektur des EU-Systems insgesamt. ${ }^{10}$

\section{Grundlegendes Dilemma des Amtes im Spiegel zweier Perspektiven}

Die inhärente Logik des hauptamtlichen Präsidenten positioniert ihn in einem idealtypischen Spannungsfeld zwischen gemeinschaftlichem Problemlösungsinstinkt einerseits und nationalem Souveränitätsreflex andererseits. ${ }^{11}$ Die Bestimmungen des Vertrages von Lissabon schaffen hierbei für den hauptamtlichen Präsidenten neue Möglichkeiten und Ermächtigungen wie auch Einschränkungen und Kontrollmechanismen in der Wahrnehmung seiner Aufgabe als Vorsitz und seiner Rolle innerhalb des Politikzyklus. Im Folgenden sollen diese durch zwei übergeordnete Orientierungsperspektiven untersucht werden.

\section{Binnenperspektive: Ermächtigungen und Einschränkungen innerhalb des Europäischen Rates als Prinzipal-Agenten-Verhältnis ${ }^{12}$}

Innerhalb des Europäischen Rates können die Staats- und Regierungschefs als kollektiver Prinzipal verstanden werden, der bestimmte Aufgaben des Vorsitzes, die sie mit der vormaligen Konfiguration nicht ausreichend erfüllt sahen, an den neuen hauptamtlichen Präsidenten, ihren Agenten, delegierten. Diese Ermächtigungen sind gleichzeitig in vertraglich festgelegte Einschränkungen und effektive prozedurale Kontrollmechanismen seines präsidialen Handlungsspielraumes eingebettet.

Die praktische Ausgestaltung des Amtes bewegt sich somit innerhalb eines weiten Rollenspektrums: So kann der Agent zu Beginn als ein ,erster Diener' vieler Herren mit stark begrenztem Spielraum für autonome Entscheidungsmacht beschrieben werden. In einer weiteren Rolle kann der Präsident auch als ,diskreter Vorsitzender', ,effektiver Manager', ,Konsensstifter' oder ,ehrlicher Makler' innerhalb seiner Institution gesehen werden, der, über Einzelinteressen der Mitgliedstaaten hinaus, Paketlösungen des Europäischen Rates in einer erweiterten Union beeinflussen und beschleunigen kann. In einer dritten Lesart kann er aber auch als ,erster Mann` oder ,erste Frau ‘13 über viele Diener gegenüber den Staats- und

9 Vgl. insbesondere die Beiträge von Wolfgang Wessels und anderen in der Sonderausgabe der integration zum Verfassungsentwurf des Europäischen Konvents. Vgl. Heinrich Schneider (Hrsg.): Der Verfassungsentwurf des Europäischen Konvents, integration 4/2003.

10 Centre for European Policy Studies/EGMONT/European Policy Centre: The Presidency of the Council: The Paradox of the New Presidency, in: Centre for European Policy Studies/EGMONT/European Policy Centre: The Treaty of Lisbon: Implementing the Institutional Innovations, Brüssel 2007, S. 41-43; Schout/Vanhoonacker: Evaluating Presidencies, 2006; Jonas Tallberg: The Power of the Presidency: Brokerage, Efficiency and Distribution in EU Negotiations, in: Journal of Common Market Studies 5/2004, S. 999-1022; Jonas Tallberg: The Agenda-Shaping Powers of the EU Council Presidency, in: Journal of European Public Policy 1/2003, S. 1-19; Fiona Hayes-Renshaw/Helen Wallace: The Council of Ministers, Basingstoke 2006.

11 Vgl. Andreas Hofmann/Wolfgang Wessels: Der Vertrag von Lissabon - eine tragfähige und abschließende Antwort auf konstitutionelle Grundfragen?, in: integration 1/2008, S. 3-20.

$12 \mathrm{Zu}$ diesem Ansatz siehe u.a. Hussein Kassim/Anand Menon: The principal-agent approach and the study of the European Union: promise unfulfilled?, in: Journal of European Public Policy 1/2003, S. 121-139; Mark A. Pollack: The Engines of European Integration. Delegation, Agency, and Agenda Setting in the EU, Oxford 2003; Mark A. Pollack: Delegation, agency, and agenda setting in the European Community, in: International Organization 1/1997, S. 99-134.

13 Zur vereinfachten Lesbarkeit und in Anlehnung an den Vertragstext wird im Folgenden nur die männliche Form genutzt. 
Regierungschefs, als ,Herr' des Verfahrens oder gar als vollwertiger ,Präsident ' mit der vollen Verantwortung einer Führungsperson gegenüber den Staats- und Regierungschefs verstanden werden. Der erste Amtsinhaber hat an verschiedenen Stellen sein Verständnis des neuen Amtes wie folgt formuliert: „Die zwei extremen Rollenmodelle sollten verworfen werden: weder ist der hauptamtliche Präsident als Präsident [im französischen Sinne] noch als einfacher Vorsitzender beabsichtigt." ${ }^{\text {"14 }}$

\section{Systemperspektive: Gleichgewicht innerhalb der EU-Architektur}

Eine zweite Systemperspektive betrifft das Gleichgewicht zwischen dem Europäischen Rat und den weiteren Institutionen der EU-Architektur. Diese unterscheidet hierbei zwischen der traditionellen intergouvernementalen und der supranationalen Ausrichtung des politischen Systems insgesamt. Einige Vertreter großer Staaten und der Präsident des Europäischen Konvents Valéry Giscard d'Estaing forderten eine Stärkung der intergouvernementalen Elemente der Europäischen Union, nicht zuletzt in Bereichen der ,high politics“.15 Kleinere Mitgliedstaaten, in der Literatur auch als „Freunde der Gemeinschaftsmethode" 16 bezeichnet, brachten seit Beginn der Verhandlungen ihre Befürchtungen zum Ausdruck, ein hauptamtlicher Präsident des Europäischen Rates könne als „Marionette“ eines Direktoriums übermäßig im Interesse großer Mitgliedstaaten handeln. ${ }^{17}$ Sie versuchten, durch die neuen Vertragsbestimmungen ihre Rolle und Interessen zu wahren, indem sie die Schwächung der Kommission als Gegengewicht zum Rat zu verhindern suchten: „Der [resultierende] Vertragstext war ein Kompromiss zwischen jenen Mitgliedstaaten, die einen starken Präsidenten nach französischem Vorbild forderten, und solchen, die eine hauptamtliche Präsidentschaft vollständig ablehnten und es vorzogen, die rotierende Präsidentschaft auf allen Ebenen zu bewahren auch weil sie eine verminderte Bedeutung der Kommission fürchteten." 18 Diese interinstitutionellen Spannungen eröffnen somit ein beträchtliches Konfliktpotenzial für die Ausführung der Rolle des Präsidenten. So bezeichnete er seine Rolle in einem kürzlich gegebenen Interview auch als „Nummer eins der wichtigsten Institution der EU“. ${ }^{19}$ Es ist folglich nicht überraschend, dass der Verdacht gegen einen zu starken oder unabhängigen Präsidenten bereits explizit in den Vertragsvorgaben zum Ausdruck kommt, nicht nur in den Modalitäten seiner Ernennung und Entlassung, sondern auch in seinem Verhältnis zu anderen Amtsinhabern.

\section{Warum ein hauptamtlicher Präsident des Europäischen Rates?}

Die rotierende Ratspräsidentschaft nimmt seit ihrer Einführung durch die Europäische Gemeinschaft für Kohle und Stahl 1951 die Rolle des Vorsitzes in den verschiedenen Rats-

14 European Council. The President: Speech by the President of the European Council, Mr. Herman Van Rompuy pronounced today at the „Klausurtagung“ of the CSU-Landesgruppe, Wildbad Kreuth, Germany, Dok. PCE 2/10, S. 5.

15 Vgl. für den Begriff Stanley Hoffmann: The State of War: Essays on the Theory and Practice of International Politics, New York 1965.

16 Lenka Rovná: Constitutionalisation. The Case of the Convention as Network Analysis, in: Lenka Rovná/Wolfgang Wessels (Hrsg.): EU Constitutionalisation: From the Convention to the Constitutional Treaty 2002-2005, Prag 2006, S. 19-49.

17 Vgl. Elmar Brok: Der Konvent - eine Chance für die Europäische Verfassung, in: integration 4/2003, S. 338344.

18 European Council. The President: Speech by the President of the European Council, 2010.

19 Klaus Jansen: Europarats-Präsident [sic!] Herman Van Rompuy, Interview mit Herman Van Rompuy, Deutsche Welle, 10.07.2010, abrufbar unter: http://www.dw-world.de/dw/article/0,,5715718,00.html (letzter Zugriff: 26.08.2010). 
formationen wahr und soll dabei eine koordinierende Funktion ausüben. Mit der Gründung des Europäischen Rates 1974 übernahm die rotierende Ratspräsidentschaft auch den Vorsitz des Europäischen Rates und somit zunehmend eine Schlüsselrolle im institutionellen Gefüge der Europäischen Union. Die Vorgaben und Ermächtigungen für diese Rolle blieben jedoch spärlich und wurden seit der Einführung der rotierenden Präsidentschaft nicht mehr vertraglich angepasst und auch nachfolgend errichteten die Mitgliedstaaten nur sehr eingeschränkte Verfahrensrechte für ihren Vorsitz.

Die Aufgaben des Europäischen Rates haben sich somit schrittweise in der gelebten Praxis weit über die geschriebenen primärrechtlichen Artikel hinaus ${ }^{20}$ entwickelt. Die so etablierten Aufgaben des Ratsvorsitzes beinhalten:

- Management und Organisation der Arbeitsweise des Europäischen Rates;

- Koordinierung der Außenpolitik auf Ebene der Staats- und Regierungschefs sowie kollektive Repräsentation der Union im internationalen Kontext;

- Förderung von Initiativen in Schlüsselbereichen und Politikfeldern als prälegislative Instanz;

- Vermittlung in Verhandlungen als ehrlicher Makler; ${ }^{21}$

- Bindeglied zwischen Regierungen und EU-Institutionen;

- transformative Führungsrolle durch langfristig angelegte Systementscheidungen zu Erweiterung und Vertiefung;

- Wächter nationaler Interessen.22

Im Lichte der Erfahrungen mit schwachen Präsidentschaften wurden strukturelle Defizite offensichtlich. ${ }^{23}$ Diese waren sowohl der kurzen Amtszeit als auch dem System der , blinden Rotation' geschuldet. Bis zum Inkrafttreten des Vertrages von Lissabon wechselte der Vorsitz des Europäischen Rates halbjährlich auf Grundlage einer durch ihn selbst bestimmten gleichberechtigten Rotation. ${ }^{24}$ Die Zeitspanne zwischen Festlegung der Präsidentschaft und dem eigentlichen präsidialen Semester eines Staats- und Regierungschefs mag somit zu suboptimalen Bedingungen für die Präsidentschaft geführt haben. Auch innenpolitische Zwänge, so zum Beispiel der Wechsel des Premierministers während der tschechischen Präsidentschaft 2009, führten zu Defiziten im alten System.

Ebenso zeigten Erfahrungen mit früheren Präsidentschaften, dass getroffene Entscheidungen oft nicht ausreichend weitergeführt wurden und der anschließende Vorsitz ganz eigenen Zielen und Prioritäten folgte. So hat zum Beispiel die französische Initiative der Union für das Mittelmeer nach der Präsidentschaft stark an Dynamik verloren. Schließlich wurden Erfolge und Misserfolge früherer Präsidentschaften nicht selten durch persönliche Ambitionen und Profile, Stile und Ressourcen sowie den historischen Kontext und aktuelle Ereignisse geprägt, in manchen Fällen auch durch exzessive Personalisierung und unproduktiven Aktionismus. ${ }^{25}$

20 Vgl. auch Hayes-Renshaw/Wallace: The Council of Ministers, 2006; Schout/Vanhoonacker: Evaluating Presidencies, 2006; Tallberg: The Power of the Presidency, 2004.

21 Schout/Vanhoonacker: Evaluating Presidencies, 2006, S. 1055.

22 Hayes-Renshaw/Wallace: The Council of Ministers, 2006, S. 140; John Peterson/Michael Shackleton: The Institutions of the European Union, 2. Auflage, Oxford 2006, S. 70.

23 Ebenda, S. 154-157.

24 Seit dem Inkrafttreten des Vertrages von Lissabon: Art. 16 Abs. 9 des Vertrages über die Europäische Union (EUV) und Art. 236 des Vertrages über die Arbeitsweise der Europäischen Union (AEUV), beide in: Amtsblatt der EU, Nr. C 83 vom 30. März 2010.

25 Centre for European Policy Studies/EGMONT/European Policy Centre: The Presidency of the Council, 2007, S. 42. 
In der Debatte um einen hauptamtlichen Präsidenten wurde die Diskontinuität als Haupthindernis zur effektiven Umsetzung der Entscheidungen dieses Schlüsselgremiums identifiziert. Erste umfangreichere Bemühungen zur Steigerung der Kontinuität und der Unterstützung weniger erfahrener Präsidentschaften mündeten in der Verknüpfung von drei Vorsitzländern, der Trio-Präsidentschaft, die mit Deutschland, Portugal und Slowenien 2007 erstmals eingeführt wurde.

Ein weiteres Argument für einen hauptamtlichen Präsidenten stellte der hohe Zeitaufwand dar, den das Amt des Präsidenten fordert. Die Erwartung an den Präsidenten beinhaltete im Sinne eines „,multiplen Bilateralismus“ ${ }^{“ 26}$ die traditionelle ,tournée des capitales` zur Vorbereitung einer Tagung des Europäischen Rates. ${ }^{27}$ Eine effektive Ausübung des Amtes in diesem Sinne war durch die parallelen nationalen Strukturen des Vorsitzenden eingeschränkt und offenbarte als Konsequenz nicht selten Defizite im Koordinierungsprozess der Staats- und Regierungschefs im Vorfeld von Europäischen Gipfeln.

Diese steigende Nachfrage nach einer Verstetigung und Professionalisierung einer Führungsrolle in der erweiterten Union, ${ }^{28}$ über den halbjährlich rotierenden formalen Vorsitz hinaus, kann in folgender Kosten-Nutzen-Analyse zusammengefasst werden (siehe Tabelle). ${ }^{29}$

Die erwarteten organisatorischen Vorteile und die verbesserte Erfüllung des Aufgabenprofils kulminierten so in der Forderung nach einem hauptamtlichen Präsidenten - auch wenn dies noch keine ,magische Formel` für Erfolg darstellen kann.

Tabelle: Kosten-Nutzen-Analyse einer Verstetigung und Professionalisierung der Führungsrolle in der erweiterten Union

\begin{tabular}{|l|}
\multicolumn{1}{|c|}{ Potenzieller Nutzen und Vorteile } \\
\hline - Kontinuität der Planung und Implementierung \\
- Wahl einer geeigneten Persönlichkeit \\
- Bildung eines politischen Vertrauenskapitals des Amtsinhabers \\
- ,Gesicht‘ und ,Stimme‘ in der Außenvertretung der Union \\
- Kein Hissen einer, nationalen Flagge‘ \\
- Umfassendes Zeitbudget \\
- Vertrauen in die Rolle eines ehrlichen Maklers \\
\hline
\end{tabular}

26 Vgl. Simon Bulmer/Wolfgang Wessels: The European Council, Decision-Making in European Politics, Houndmills 1987, S. 54-55.

27 Vgl. Generalsekretariat des Rates: Die Arbeitsweise des Rates in einer erweiterten Union, Bericht der vom Generalsekretär des Rates eingesetzten Arbeitsgruppe, SN 2139/99; Herman Van Rompuy: Speech at EMI (European Movement International), 25.05.2010, S. 3.

28 Wolfgang Wessels: The European Council: A Bigger Club, a Similar Role?, in: Edward Best/Thomas Christiansen/Pierpaolo Settembri (Hrsg.): The Institutions of the enlarged European Union. Continuity and Change, Cheltenham 2008, S. 16-33; Wolfgang Wessels: A New Top? The Lisbon Provisions for the Permanent President of the European Council, SCEUS Salzburg Centre of European Union Studies/Jean Monnet Centre of Excellence: Salzburg Papers on European Integration 2/2010.

29 Vgl. Herman Van Rompuy: Speech at EMI, 2010; Centre for European Policy Studies/EGMONT/European Policy Centre: The Presidency of the Council, 2007, S. 46-48. 


\section{Potenzielle Kosten und Nachteile}

- Aufhebung der Einheit innerhalb der Präsidentschaft - fragmentierte Weisungskette und komplexe (auch informelle) Koordinierungsverfahren

- Gefahr der Wahl einer unqualifizierten Persönlichkeit aufgrund der Politisierung des Auswahlverfahrens

- Rivalität zwischen dem Präsidenten, der rotierenden Präsidentschaft und dem Hohen Vertreter der Union für Außen- und Sicherheitspolitik: Aufweichung der Verantwortlichkeiten

- Weiterhin eingeschränkte Amtszeit (maximal fünf Jahre)

- EU-fokussierte Verengung des Blickfeldes: Verlust des Gefühls für nationale Sorgen und Sensibilitäten

- Demotivation ehrgeiziger Regierungschefs als Mitglieder des Europäischen Rates

- Keine Mobilisierung nationaler Ressourcen

Quelle: Eigene Darstellung.

\section{Der hauptamtliche Präsident in der Binnenperspektive: Ermächtigungen und Ein- schränkungen}

\section{Wahl und Amtsdauer}

Die Wahl und die Amtsdauer des Präsidenten zeigen einige Besonderheiten. „Der Europäische Rat wählt seinen Präsidenten mit qualifizierter Mehrheit“ ". ${ }^{30}$ Im Gegensatz zum Präsidenten der Kommission und der Hohen Vertreterin der Union für Außen- und Sicherheitspolitik ist der hauptamtliche Präsident trotz des Anspruches an gesteigerte Kontinuität nur für zweieinhalb Jahre gewählt und sein Mandat ist insgesamt auf zwei Amtszeiten oder insgesamt fünf Jahre beschränkt. Als effektiver Kontrollmechanismus kann die Möglichkeit der Entlassung des Präsidenten des Europäischen Rates gewertet werden. „Im Falle einer Verhinderung oder einer schweren Verfehlung kann der Europäische Rat ihn auf dem Wege des gleichen Verfahrens [der qualifizierten Mehrheit] von seinem Amt entbinden. " ${ }^{31}$ Diese Regelungen werden den Präsidenten durch eine Form des ,vorauseilenden Gehorsams ' gegenüber einer ,Sperrminorität bei einer Wiederwahl in kontroversen Fragen und Sachentscheidungen stärker einbinden und seine persönliche Rolle in den Hintergrund drängen, indem Konsensformulierungen mit einer potenziell blockierenden Minderheit weiter gesucht oder ganz von der Tagesordnung gestrichen werden. Somit ist eine weithin von kleineren Mitgliedstaaten befürchtete dauerhafte Allianz des hauptamtlichen Präsidenten mit den drei großen Mitgliedstaaten praktisch ausgeschlossen und gleichzeitig wurde eine Option des ,impeachment light', als ultima ratio, geschaffen.

\section{Initiator und Organisator}

Art. 15 Abs. 6 EUV definiert ausführlich die Aufgaben und Funktionen des Vorsitzes und seine Rolle innerhalb des Politikzyklus: „Der Präsident des Europäischen Rates a) führt den Vorsitz bei den Arbeiten des Europäischen Rates und gibt ihnen Impulse, b) sorgt in Zusammenarbeit mit dem Präsidenten der Kommission auf der Grundlage der Arbeiten des Rates „Allgemeine Angelegenheiten“ für die Vorbereitung und Kontinuität der Arbeiten des Europäischen Rates, c) wirkt darauf hin, dass Zusammenhalt und Konsens im Europäischen Rat gefördert werden, d) legt dem Europäischen Parlament im Anschluss an jede Tagung des Europäischen Rates einen Bericht vor." Auch die organisatorische Verantwortung des Präsi-

30 Art. 15 Abs. 5 S. 1 EUV.

31 Art. 15 Abs. 5 S. 2 EUV. 
denten ist eine seiner zentralen Aufgaben, die durch die Geschäftsordnung noch einmal bestätigt wird: „Der Präsident sorgt für [...] den ordnungsgemäßen Ablauf der Arbeiten.“32

Als erster konkreter Schritt wird der „Europäische Rat [...] von seinem Präsidenten“ ,33 „,spätestens ein Jahr vor Beginn jedes Halbjahres“ und ,in enger Zusammenarbeit“ mit der rotierenden Präsidentschaft ${ }^{34}$ einberufen. „,[]]n Zusammenarbeit mit dem Präsidenten der Kommission [und] auf der Grundlage der Arbeiten des Rates , Allgemeine Angelegenheiten “ [sorgt er] für die Vorbereitung und Kontinuität der Arbeiten des Europäischen Rates“ ${ }^{35}$ Er legt mit der rotierenden Präsidentschaft und dem Kommissionspräsidenten ,dem Rat ,Allgemeine Angelegenheiten" mindestens vier Wochen vor jeder ordentlichen Tagung [...] einen Entwurf einer erläuterten Tagesordnung vor." ${ }^{36}$ Fünf Tage vor der Tagung erstellt der Präsident die vorläufige Tagesordnung auf der Grundlage der letzten Aussprache des Rates ,Allgemeine Angelegenheiten“. Innerhalb des Politikzyklus kann der Präsident die Debatte um Tagesordnungspunkte somit bereits im Vorfeld anregen und anleiten. Er unterbreitet ebenso einen „Entwurf von Leitlinien für die Schlussfolgerungen des Europäischen Rates und gegebenenfalls die Entwürfe von Schlussfolgerungen und die Entwürfe von Beschlüssen des Europäischen Rates“. 37 Auch sind die Tagungen des Europäischen Rates eng mit den anderen Akteuren vorzubereiten. Die Geschäftsordnung betont erneut eine ,enge [interinstitutionelle] Zusammenarbeit und Abstimmung“, ,insbesondere durch regelmäßige Treffen“ „,mit dem Vorsitz des Rates und dem Präsidenten der Kommission" ". ${ }^{38}$ Weiterhin ist die Einflussnahme weiterer Ratsformationen auf diese Zusammenarbeit eingeschränkt: „Außer aus zwingendem und unvorhergesehenem Anlass, z. B. im Zusammenhang mit dem internationalen Tagesgeschehen, darf keine andere Ratsformation und kein anderes Vorbereitungsgremium zwischen der Tagung des Rates, Allgemeine Angelegenheiten', auf der die vorläufige Tagesordnung des Europäischen Rates erstellt wird, und der Tagung des Europäischen Rates ein Thema erörtern, das dem Europäischen Rat unterbreitet wird“. ${ }^{39}$ Durch diese „enge Zusammenarbeit" sieht die Geschäftsordnung folglich eine Funktion des Präsidenten als ,gate keeper' vor, die weder durch den Kommissionspräsidenten noch den Vorsitz der rotierenden Präsidentschaft umgangen werden kann.

\section{Sitzungsleiter}

Wie in anderen Gremien auch verlangt der Ablauf der Tagungen nach Führung im Sinne eines „Organisators“40 oder eines ,,business manager“ ${ }^{41}$ Bereits in den Regeln für die Organisation der Beratungen des Europäischen Rates in der Erklärung von Sevilla ist für den Präsidenten vorgesehen, dass er für ,einen ordnungsgemäßen Ablauf der Beratungen“ sorgt und Maßnahmen ergreift, die eine ,optimale Nutzung der verfügbaren Zeit“ fördern, so zum Beispiel ,die Festlegung der Reihenfolge, in der die Tagesordnungspunkte behandelt werden, die Begrenzung der Redezeit oder die Festlegung der Reihenfolge der Redebeiträge. ${ }^{42}$ Sie

32 Art. 4 Abs. 4 der Geschäftsordnung des Europäischen Rates (Geschäftsordnung). Vgl. Beschluss des Europäischen Rates zur Festlegung seiner Geschäftsordnung, 2009.

33 Art. 15 Abs. 3 EUV.

34 Art. 1 Abs. 1 Geschäftsordnung.

35 Art. 15 Abs. 6 Lit. b EUV.

36 Art. 3 Abs. 1 Geschäftsordnung.

37 Ebenda.

38 Art. 2 Abs. 3 Geschäftsordnung.

39 Art. 3 Abs. 2 Geschäftsordnung.

40 Vgl. Schout/Vanhoonacker: Evaluating Presidencies, 2006, S. 1053-1056.

41 Vgl. Hayes-Renshaw/Wallace: The Council of Ministers, 2006, S. 141-144.

42 Rat der Europäischen Union: Europäischer Rat (Sevilla) Tagung vom 21. und 22. Juni 2002. Schlussfolgerungen des Vorsitzes, Dok. 13463/02. 
ermöglichen dem Präsidenten auch, Sitzungen zu unterbrechen um auf einen Konsens hinzuwirken. Er kann ebenso die Änderung der Tagungsdauer durch den Europäischen Rat oder den Rat „Allgemeine Angelegenheiten” veranlassen. ${ }^{43}$ Auch könnte der Präsident ,entscheiden, dass eine Tagung des Europäischen Rates an einem anderen Ort [als Brüssel] abgehalten wird“ wenn der Rat „Allgemeine Angelegenheiten“ oder der Ausschuss der Ständigen Vertreter dies einstimmig fordert. ${ }^{44}$

Diese Verfahrenskompetenz als Vorsitzender stattet das Amt des Präsidenten folglich mit einem gewissen Grad an Autorität aus. Diese Regeln implizieren oder verlangen, dass der Präsident den Verlauf der Diskussionen maßgeblich leitet und erlauben ihm ein gewisses $\mathrm{Ma} ß$ an Flexibilität. Aus der Prinzipal-Agenten-Perspektive ist ihm somit eine gestärkte Rolle zugewiesen worden und die Mitglieder des Europäischen Rates haben sich bereit erklärt, dem organisatorischen Können ihres Präsidenten in den Beratungen zu folgen. Im Spannungsfeld unterschiedlicher Erwartungen, persönlichem Engagements und geforderter Diskretion muss er in der gelebten Praxis seinen eigenen Stil entwickeln. Wahrgenommene Erfolge in diesem Amt können das politische Kapital des Amtsinhabers stärken, Misserfolge oder übermäßiger Ehrgeiz und Führungsanspruch gegenüber seinen ,peers‘ jedoch auch schwächen.

\section{Konsens-Manager - ehrlicher Makler}

Das grundlegende Entscheidungsprinzip des Europäischen Rates bleibt die Einstimmigkeit: ,Soweit in den Verträgen nichts anderes festgelegt ist, entscheidet der Europäische Rat im Konsens"“. ${ }^{45}$ Trotz einer gewissen Manövrierfähigkeit bleibt der Präsident in all seinen Aussagen und Erklärungen zur Union somit stets strukturell an das Erfordernis der gemeinsamen Position aller Mitglieder gebunden. Dieses Prinzip zeigt am deutlichsten die Rolle des ,Dieners', des , ausführenden Agenten“ oder gar nur ,Sprechers' der Staats- und Regierungschefs. Es ist ebenso Ausdruck und Garant der Interessenwahrung einzelner, besonders kleinerer, Staaten im Gleichgewicht mit größeren Mitgliedstaaten innerhalb des Europäischen Rates.

In Ausübung dieser Funktion als Konsensmakler soll der Präsident deliberative und argumentative Verhandlungen fördern und dabei verschiedene Mittel und Taktiken einsetzen. Ein interessantes Instrument ist besonders das „Beichtstuhlverfahren“:46 Außerhalb des offenen Plenums lotet der Präsident mit den betroffenen Mitgliedern Präferenzen, Minimalforderungen und ,rote Linien ‘ dieser Protagonisten aus und kann unmittelbaren Druck ausüben, vorgefertigte Positionen neu auszutarieren.

Wenn davon ausgegangen wird, dass das deutsch-französische Tandem eine zentrale Rolle im Europäischen Rat spielt, ergibt sich für den Präsidenten insbesondere die Schwierigkeit, Kompromisse zwischen den merklich unterschiedlichen Positionen beider Staaten herbeizuführen und dabei die potenzielle Dynamik einer ,,wohlwollenden Hegemonie“477 zu nutzen, ohne den anderen Mitgliedern den Eindruck eines Diktats zu vermitteln oder selbst als Marionette des Duos wahrgenommen zu werden. In der Rolle des geschickten Mediators mag sich der Präsident auch neu gestalteter Formulierungen bedienen, die aufgrund gewisser

43 Art. 4 Abs. 1 Geschäftsordnung.

44 Art. 1 Abs. 2 Geschäftsordnung.

45 Art. 15 Abs. 4 EUV.

46 Hayes-Renshaw/Wallace: The Council of Ministers, 2006, S. 150.

47 William Paterson: Does Germany still have a European vocation?, in: German Politics 1/2010, S. 41-52. 
Zweideutigkeiten und Unschärfen, Auslassungen und Vertagungen schließlich einen Konsens ermöglichen.

In bestimmten Fällen, zum Beispiel bei der Wahl von Personen für Spitzenämter in EUInstitutionen, sieht Artikel 6 der Geschäftsordnung ein besonderes Verfahrensrecht vor: „In den Fällen, in denen der Europäische Rat im Einklang mit den Verträgen einen Beschluss im Rahmen einer Abstimmung annimmt, erfolgt die Abstimmung auf Veranlassung seines Präsidenten. “48 Durch dieses Verfahren ist es dem Präsidenten möglich, durch die explizite Abstimmung hartnäckige Vertreter von Minderheitspositionen zu Kompromissen zu bewegen ähnlich den etablierten und häufig wiederkehrenden Verhaltensmustern im Ministerrat. ${ }^{49}$ Auch bei Nichtausübung dieses Rechts entfaltet es doch eine gewisse disziplinierende Wirkung, die die Rolle des Präsidenten als ehrlichen Makler stärken kann. Ein weiteres Verfahrensrecht ermöglicht dem Präsidenten gemeinsame Entscheidungen voranzutreiben: „Beschlüsse des Europäischen Rates über eine dringende Angelegenheit können durch schriftliche Abstimmung angenommen werden, wenn der Präsident des Europäischen Rates die Anwendung dieses Verfahrens vorschlägt [...] [und] sich alle Mitglieder [...] einverstanden erklären." ${ }^{50}$

Im Vergleich zu Ratspräsidentschaften der ressourcenstarken Mitgliedstaaten verfügt der hauptamtliche Präsident in Verhandlungen jedoch nur über eingeschränkte institutionelle Möglichkeiten und weitaus weniger Ressourcen, um Angebote zur Überwindung einer Pattsituation in einem Gesamtpaket zu unterbreiten. Das Motto ,Präsidentschaft verpflichtet" ist ohne Anreizmechanismen für unwillige Mitglieder weitgehend entkräftet. Aufgrund dieses fehlenden Moments befürchten Skeptiker, dass der, entnationalisierte' Amtsinhaber die implizite Forderung nach Führungsstärke nur begrenzt ausführen können wird. So wird der Präsident des Europäischen Rates auch in möglichen Auseinandersetzungen zwischen Regierungschefs und dem Kommissionspräsidenten vermitteln müssen, entlang einer potenziell konfliktträchtigen Trennlinie zwischen mitgliedstaatlichen und Unionsinteressen. Im Laufe von fünf aufeinanderfolgenden rotierenden Präsidentschaften in seiner Amtszeit oder möglichen zehn bei einer Wiederwahl mag der Präsident dennoch ein enges Netzwerk persönlicher Kontakte aufbauen, das sich besonders in Krisenzeiten als äußerst nützlich erweisen kann. Erste Hinweise scheinen die Formierung einer solchen Führungsposition mit zunehmend zentraler Bedeutung noch nicht zu bestätigen. ${ }^{51}$ Die reale Nutzung (oder NichtNutzung) der Möglichkeiten durch Herman Van Rompuy bleibt somit weiterhin ein genau zu beobachtender Faktor.

\section{Außenvertreter der Union}

Im Bereich der Gemeinsamen Außen- und Sicherheitspolitik der Union haben die Staatsund Regierungschefs im Laufe der Zeit der Präsidentschaft auch die Aufgabe der Vertretung der Union im internationalen System auf höchster Ebene zugewiesen. ${ }^{52}$ Der Vertrag von Lissabon hat nun die Rolle des Europäischen Rates im ,auswärtigen Handeln der Union“ als

48 Art. 6 Abs. 2 Geschäftsordnung.

49 Hayes-Renshaw/Wallace: The Council of Ministers, 2006, S. 147-149.

50 Art. 7 Geschäftsordnung.

51 Daniela Kietz/Nicolai von Ondarza: Willkommen in der Lissabonner Wirklichkeit, Stiftung Wissenschaft und Politik: SWP-Aktuell 29, Berlin 2010.

52 Während der deutschen Ratspräsidentschaft im ersten Halbjahr 2007 führte die Kanzlerin den Vorsitz von vier bilateralen EU-Gipfeltreffen (mit den USA, Kanada, Japan und Russland) sowie zwei Tagungen des Europäischen Rates und der Feier zum 50-jährigen Bestehen der Römischen Verträge. 
Institution insgesamt aufgewertet, indem er ihm die Festlegung der „strategischen Interessen und Ziele der Union" für das gesamte Politikfeld zuschreibt. ${ }^{53}$

Aber auch hier zogen die Staats- und Regierungschefs klare Trennlinien: So vertritt der hauptamtliche Präsident die Union nur in Fragen der Außen- und Sicherheitspolitik, ,auf seiner Ebene und in seiner Eigenschaft, unbeschadet der Befugnisse des Hohen Vertreters der Union für Außen- und Sicherheitspolitik“"54 und er darf sich gemeinsam mit der Hohen Vertreterin der Union für Außen- und Sicherheitspolitik des ebenfalls neu geschaffenen Europäischen Auswärtigen Dienstes bedienen. ${ }^{55}$ Nach dem Verständnis des neuen Amtsinhabers ist „,der hauptamtliche Präsident die Person, die Staats- und Regierungschefs aus Drittländern im Namen der Europäischen Union empfängt und besucht. "56 Während manche dieser Aufgaben protokollarischer Natur sind, hat sich Van Rompuy durch seinen Anspruch auf eine Spitzenposition doch in ein mögliches Spannungsfeld mit der Hohen Vertreterin der Union für Außen- und Sicherheitspolitik gerückt, das gegenwärtig jedoch als nicht problematisch erachtet werden muss.

Darüber hinaus birgt der Bereich des auswärtigen Handelns der Union besonderes Potenzial für Konfrontationen mit den Interessen nationaler Entscheidungszentren, besonders denen größerer Mitgliedstaaten. Es ist zu erwarten, dass nationale Regierungschefs, als kollektiver Prinzipal, ,ihrem‘ Präsidenten nur zögerlich eine tragende oder gar exklusive Rolle zuteil werden lassen, wenn sie den Auftritt auf dem internationalen Parkett für sich selbst beanspruchen oder als angemessen betrachten. Um diese generell vorsichtige Einstellung der Mitgliedstaaten zu dokumentieren, betonen die angehängten Erklärungen 13 (und 14) der Schlussakte der Regierungskonferenz von Lissabon, dass die Bestimmungen des Vertrages von Lissabon „weder die derzeit bestehenden Zuständigkeiten der Mitgliedstaaten für die Formulierung und Durchführung ihrer Außenpolitik noch ihre nationale Vertretung in Drittländern und internationalen Organisationen berühren". ${ }^{57}$ Obgleich sich beide Texte auf die Rolle der Hohen Vertreterin der Union für Außen- und Sicherheitspolitik und den neuen Auswärtigen Dienst beziehen, sind sie doch Ausdruck einer grundsätzlichen Haltung, die dem Geiste nach als eine dem Abkommen von Luxemburg (1966) analoge Logik interpretiert werden kann, das den Präsidenten des Europäischen Rates in das enge und berechnende Korsett sehr wichtiger nationaler Interessen eines oder mehrerer Partner schnürt.

Konsultationen in allen EU-Hauptstädten, die Vertretung der Union in bisher über 60 bilateralen Gipfeln ${ }^{58}$ (teilweise gemeinsam mit dem Kommissionspräsidenten) und bilaterale Treffen mit Regierungschefs von Drittstaaten seit dem Amtsantritt des Präsidenten deuten auf eine pro-aktive Auslotung seiner Möglichkeiten hin. Beratung und Koordinierung des auswärtigen Handelns müssen somit zwischen dem hauptamtlichen Präsidenten und der Hohen Vertreterin der Union für Außen- und Sicherheitspolitik stattfinden, aber auch mit dem rotierenden Vorsitz und dem Kommissionspräsidenten (siehe unten) sowie gegebenenfalls auch mit dem Präsidenten der Euro-Gruppe. ${ }^{59}$ Obwohl die neue institutionelle Ausgestal-

53 Art. 22 Abs. 1 und Art. 24 EUV.

54 Art. 15 Abs. 6 EUV.

55 Julia Lieb/Martin Kremer: Der Aufbau des Europäischen Auswärtigen Dienstes: Stand und Perspektiven, in: integration 3/2010, S. 195-208.

56 Siehe Van Rompuy: Speech at EMI, 2010.

57 13. Erklärung zur Gemeinsamen Außen- und Sicherheitspolitik, in: Amtsblatt der EU, Nr. C 83 vom 30. März 2010, S. 343. Siehe auch 14. Erklärung zur Gemeinsamen Außen- und Sicherheitspolitik, in: Amtsblatt der EU, Nr. C 83 vom 30. März 2010, S. 343.

58 Eigene Zählung (seit Amtsantritt).

59 Art. 2 des Protokolls Nr. 14 betreffend die Euro-Gruppe, in: Amtsblatt der EU, Nr. C 83 vom 30. März 2010, S. 283. 
tung der Vereinfachung der auswärtigen Vertretung dienen sollte, ist „die Situation eher kompliziert und komplex geworden" " ${ }^{60}$ Die spärlichen primärrechtlichen Vorgaben beinhalten keine abschließend klärenden Aufgabenzuweisungen zwischen den für die Außenvertretung zuständigen Ämtern der Europäischen Union und riskieren in Anbetracht der Forderung einer gemeinsamen und einheitlichen Stimme Europas in der Welt als ein weiteres „capability-expectation gap“61 wahrgenommen zu werden.

\section{Krisenmanager}

In Fällen akuter Krisen haben Politiker und Medien ihr Augenmerk ebenfalls verstärkt auf den Europäischen Rat und besonders seinen Präsidenten gerichtet. Der neue Amtsinhaber sieht sich mit der Erwartung konfrontiert, dass seine Institution ausreichend auf Schwierigkeiten der Union im internationalen Umfeld reagieren kann. Als Verfahrensinstrument zur Durchführung eines solchen Krisenmanagements, wenn dies erforderlich ist, ,beruft der Präsident eine außerordentliche Tagung des Europäischen Rates ein“.62 Er verfügt damit über das Recht, kurzfristig, Notfallgipfel` abzuhalten oder in relevanten Fällen auch auf das schriftliche Verfahren zur Erlangung eines gemeinsamen Standpunktes zurückzugreifen (siehe oben).

Eine wichtige Priorität auf der Tagesordnung des Präsidenten war und ist die Finanz- und Euro-Krise. Präsident Van Rompuy organisierte über die normalen Tagungen hinaus auch informelle Treffen und Arbeitsgruppen des Europäischen Rates außerhalb des Ratsgebäudes. Dies deutet zunächst auf eine aktive Unterstützung und Akzeptanz des Präsidenten in dieser Rolle unter den Mitgliedstaaten hin. Die Staats- und Regierungschefs der Mitgliedstaaten der Euro-Gruppe haben ihn ebenso mit dem Vorsitz der Arbeitsgruppe „Wirtschaftspolitische Steuerung“ beauftragt, der die 27 zuständigen Minister der Mitgliedstaaten sowie der Kommissar für Wirtschaft und Währung, der Präsident der Europäischen Zentralbank und der Präsident der Euro-Gruppe angehören.

\section{Der Präsident des Europäischen Rates im Gleichgewicht der institutionellen EU-Ar- chitektur}

Im Sinne der Systemperspektive stellt sich die Frage nach dem Einfluss des neuen Präsidenten auf das institutionelle Gleichgewicht innerhalb des EU-Systems. Die Bestimmungen für den Europäischen Rat und seinen Präsidenten wurden in einem größeren politischen Paket geschnürt, das ein neues institutionelles Gleichgewicht , auf höherer Ebene ' errichten sollte: durch intergouvernementale und ,,souveränitätsschonende“63 Zugeständnisse an die Mitgliedstaaten wie auch föderaler und supranationaler Schutzmechanismen, zum Beispiel die Kontrolle verbindlicher Entscheidungen des Europäischen Rates durch den Gerichtshof. Der Präsident selbst kann dabei eine aktive Führungsrolle forcieren (,push') oder angetragen (,pull') bekommen, so im frühen Stadium der Beratungen über System- und Politikgestaltung im Sinne der prälegislativen und verfassungsgebenden Bestrebungen des Europäischen

60 Siehe Van Rompuy: Speech at EMI, 2010.

61 Siehe für den Begriff Christopher Hill: The Capability-Expectations Gap, or Conceptualizing Europe's International Role, in: Journal of Common Market Studies 3/1993, S. 305-328.

62 Art. 15 Abs. 3 EUV.

63 Vgl. Centre for European Policy Studies/EGMONT/European Policy Centre: The Presidency of the Council, 2007, S. 49; Hofmann/Wessels: Der Vertrag von Lissabon - eine tragfähige und abschließende Antwort, 2008; Brigid Laffan: Getting to a European Constitution: From Fischer to the IGC, in: Sonja Puntscher Riekmann/ Wolfgang Wessels (Hrsg.): The Making of a European Constitution. Dynamics and Limits of the Convention Experience, Wiesbaden 2006, S. 68-89, hier S. 76. 
Rates. Er kann dies zum Beispiel durch die Einsetzung und den Vorsitz von Arbeitsgruppen, einem beliebten Mittel des Europäischen Rates, oder durch Grundsatzreden fördern. Im Sinne der Prinzipal-Agenten-Analyse kann der Präsident auch als ,Agent ‘ für die weitere institutionelle Architektur der Europäischen Union verstanden werden, nicht nur um den Europäischen Rat zu führen, sondern auch um andere EU-Institutionen, insbesondere die Europäische Kommission, die Hohe Vertreterin der Union für Außen- und Sicherheitspolitik anzuleiten.

\section{Beziehungen zur rotierenden Ratspräsidentschaft und zum Rat}

Im Verhältnis zur rotierenden Ratspräsidentschaft kann eine mögliche Rivalität zweier Präsidenten eine demotivierende Wirkung entfalten, wenn hierdurch die nationale Bereitschaft, Ressourcen und persönliche Reputation für die Gemeinschaftsaufgaben einzubringen, verringert würde. Möglich ist indes auch der umgekehrte Fall. Die belgische Regierung hat mit Übernahme der Ratspräsidentschaft im Juli 2010 bereits angekündigt, ihre Aufgaben eng mit dem Präsidenten des Europäischen Rates abzustimmen. Sie begründet dies mit der Umsetzung des Vertrages von Lissabon, so zum Beispiel im Sinne des Art. 4 Abs. 1 der Geschäftsordnung: „Das Mitglied des Europäischen Rates, das den Mitgliedstaat vertritt, der den Vorsitz des Rates wahrnimmt, erstattet dem Europäischen Rat im Benehmen mit dessen Präsidenten Bericht über die Arbeiten des Rates." Es ist eine besondere Koinzidenz, dass die durch innenpolitische Konflikte geschwächte geschäftsführende Regierung Belgiens nun mit ihrem früheren und vergleichsweise starken Premier Van Rompuy in dieser Konstellation zusammenarbeitet. Gerade weil das neue Amt noch reichlich Spielraum zur Profilbildung lässt und neue Formen der praktischen Zusammenarbeit und informeller Prozesse verlangt, könnte dies verstärkt zur Einführung ,kurzer Dienstwege' führen, die das Verhältnis zwischen rotierendem und hauptamtlichem Vorsitz auch in Zukunft nachhaltig prägen werden.

Skeptiker argumentieren, dass in dem , gemischten Präsidialkonzept ${ }^{664}$ die tradierten Kommunikationslinien, insbesondere zwischen der Präsidentschaft des Europäischen Rates und dem Rat „Allgemeine Angelegenheiten“ zur Sicherstellung und Weiterführung von Beschlüssen, ${ }^{65}$ ausgehebelt werden könnten. So hat der erste Amtsinhaber eine gewisse „Abwesenheit organischer Verknüpfungen zwischen dem Europäischen Rat und den Fachräten [auch] als nennenswertes Hindernis" 66 bezeichnet. Formal bleiben die vertraglich vorgesehenen legislativen Möglichkeiten des Europäischen Rates zwar eingeschränkt und institutionell eingebunden. Er wird weiterhin ,nicht gesetzgeberisch tätig." ${ }^{67}$ Besonders jedoch gegenüber dem Rat „Allgemeine Angelegenheiten“, wie auch je nach Sachgebiet den weiteren Ratsformationen, so zum Beispiel dem Rat „Wirtschaft und Finanzen“ (ECOFIN), bleibt zu beobachten, inwiefern die gestärkte Rolle des Europäischen Rates und seines Präsidenten eine mögliche ,schleichende Intergouvernementalisierung' der Entscheidungsfindung und des politischen Systems nach sich ziehen könnte.

Insgesamt entsteht durch die veränderte Konfiguration die Gefahr neuer potenzieller Rivalitäten, die sich durch eine unklare Verteilung von Zuständigkeiten ausweiten können: im Verhältnis zum Regierungschef der rotierenden Präsidentschaft und dem Team der Trio-Präsi-

64 Vgl. auch Centre for European Policy Studies/EGMONT/European Policy Centre: The Presidency of the Council, 2007.

65 Art. 16 Abs. 6 EUV.

66 Siehe Van Rompuy: Speech at EMI, 2010.

67 Art. 15 Abs. 1 EUV. 
dentschaft, der Hohen Vertreterin der Union für Außen- und Sicherheitspolitik - insbesondere in ihrer Rolle als Vorsitzende des Rates „Auswärtige Angelegenheiten“-, dem intern gefestigten Amt des Kommissionspräsidenten, dem Präsidenten eines mächtigeren Europäischen Parlamentes und potenziell auch dem nun rechtlich verankerten Präsidenten der Euro-Gruppe.

\section{Kommission und Kommissionspräsident}

Die rotierende Präsidentschaft war ursprünglich in ein institutionelles Gleichgewicht eingebettet, in dem die Kommission als Monopolistin des Initiativrechts eine starke Rolle innehatte ${ }^{68}$ - im Sinne eines Tandems zwischen Kommission, die vorzuschlagen, und des Rates, der zu entscheiden hat. ${ }^{69}$ Die Architekten der ursprünglichen Verträge vergaben die Rolle des Vorsitzes somit an einen nationalen Minister, um einer Machtkonzentration der supranationalen Institutionen vorzubeugen.

In den Debatten um den Vertrag von Lissabon wurden nun Bedenken geäußert, die Kommission könne an Einfluss verlieren und durch die Dominanz eines neuen hauptamtlichen Präsidenten ihre Schutzfunktion für kleinere Mitgliedstaaten gegen eine Maschinerie größerer Mitgliedstaaten einbüßen. Die Vorgaben des Vertrages sehen jedoch ausdrücklich eine Rolle des Kommissionspräsidenten innerhalb des Europäischen Rates vor, die die Autonomie des hauptamtlichen Präsidenten einbindet. So sind Vorbereitungen und Arbeiten der Institution nur ,in Zusammenarbeit mit dem Präsidenten der Kommission [und] auf der Grundlage der Arbeiten des Rates ,Allgemeine Angelegenheiten“"70 durchzuführen. Ohne die Unterstützung und Einwilligung des Kommissionspräsidenten - sowie auch das Mandat durch den Rat „Allgemeine Angelegenheiten“- bleibt der hauptamtliche Vorsitz nur eingeschränkt handlungsfähig.

Auch in der Außenvertretung behält der Kommissionspräsident sein Vorrecht, die Union in allen anderen Belangen als der Gemeinsamen Außen- und Sicherheitspolitik gegenüber Drittstaaten zu vertreten. Jedoch gelang es dem hauptamtlichen Präsidenten bereits bei verschiedenen Anlässen, wie zum Beispiel dem EU-Russland-Gipfel 2010, seine Position und Sichtbarkeit gegenüber dem Kommissionspräsidenten, auch medial, effektiv zu behaupten. Ein gewisser Eindruck des Ringens um Aufmerksamkeit zwischen den Ämtern bleibt indes also nicht aus und wird durch so verstandene Versuche der Aufwertung oder Personalisierung einer Führungsrolle auf beiden Seiten weiter genährt, so zum Beispiel durch die kürzliche ,State of the Union'-Rede des Kommissionspräsidenten José Manuel Barroso vor dem Europäischen Parlament sowie durch Videonachrichten und online archivierte Reden des Präsidenten des Europäischen Rates.

In der Praxis des ersten Halbjahres der Präsidentschaft Van Rompuys deutet sich mit dem Kommissionspräsidenten jedoch eine Form der, cohabitation ${ }^{71}$ an, die sich zum Beispiel in Form des wöchentlichen Gedankenaustausches zwischen Barroso und Van Rompuy eingespielt hat. Zusammen mit den weiteren Präsidenten innerhalb der Unionsarchitektur kann diese Konstellation nach einer Anfangsphase der dynamischen Rivalität in eine gemeinsame informelle Wahrnehmung kollektiver Führungsaufgaben ${ }^{72}$ übergehen.

68 Vgl. Kenneth A. Sheplse: Institutional Equilibrium and Equilibrium Institutions, in: Herbert F. Weisberg (Hrsg.): Political Science: The Science of Politics, New York 1986, S. 51-82.

69 Vgl. Udo Diedrichs/Wolfgang Wessels: The Commission and the Council, in: David Spence/Geoffrey Edwards (Hrsg.): The European Commission, London 2006, S. 209-234.

70 Hervorhebung durch die Autoren. Art. 15 Abs. 6 Lit. b EUV.

71 Vgl. Ben Crum: Accountability and Personalisation of the European Council Presidency, in: Journal of European Integration 6/2009, S. 685-701.

72 Siehe Jack Hayward: Leaderless Europe, Oxford 2008. 


\section{Europäisches Parlament}

Zur Steigerung der Transparenz ist der Präsident des Europäischen Rates in Fortführung etablierter Zusammenarbeit zur Berichterstattung und Rechenschaftspflicht vor dem Europäischen Parlament vertraglich angehalten. ${ }^{73}$ In der Vergangenheit war das Europäische Parlament mit der Form der Unterrichtung in den Plenarsitzungen nicht selten unzufrieden, da Präsidentschaften oft die Presseberichte wiederholten, die sie bereits unmittelbar im Anschluss an ihre Sitzungen veröffentlicht hatten. Der neue hauptamtliche Präsident bemüht sich daher, die wenigen Regeln im Zusammenspiel mit dem Europäischen Parlament ,korrekt zu interpretieren “. ${ }^{74}$ Regelmäßige Treffen mit dem Präsidenten des Europäischen Parlamentes und ,briefings“ mit Arbeitsgruppen im Anschluss an Tagungen des Europäischen Rates finden in der Praxis bereits statt.

Insbesondere Mitglieder des Europäischen Parlamentes äußerten in den Debatten um den neuen Vertrag die Besorgnis, der neue Präsident könne als ,Diener' oder ,Sprecher ' für ein Direktorium großer Staaten zu Zwecken der Herrschaft , von oben " missbraucht werden. ${ }^{75}$ Mit der rotierenden Präsidentschaft auf Ministerebene im Rat und der Präsidentschaft auf Spitzenebene seien mögliche Tendenzen und Risiken ,,autokratischen Verhaltens" 76 genauestens zu beobachten. Der Vorschlag von Giscard d'Estaing im Europäischen Konvent, den hauptamtlichen Präsidenten zukünftig durch die Wahl europäischer und nationaler Parlamentarier unabhängig und gleichsam bürgernah einzusetzen, wurde jedoch von den Prinzipalen nicht weiterverfolgt.

\section{Schlussfolgerungen: ,Herr' oder ,erster Diener‘?}

In der Schaffung des neuen Amtes des hauptamtlichen Präsidenten des Europäischen Rates sind die Staats- und Regierungschefs der Mitgliedstaaten als Prinzipale den möglichen Gefahren unbeabsichtigter Konsequenzen oder eines ,drift' ihres Agenten vorausschauend begegnet. Aus einer Binnenperspektive haben sie ihn innerhalb ihrer Institution mit eingeschränkten Verfahrensrechten ausgestattet und in ein komplexes mehrschichtiges Kontrollgeflecht eingebettet, das eine Verselbständigung ihres Präsidenten verhindern soll. Neben den ersten konkreten Bestimmungen zu Wahl, Entlassung und Amtszeit des Präsidenten bilden diese Regeln ein Sicherheitsnetz zur Machtbegrenzung des Amtes. Vor dem Hintergrund der Erfahrungen mit der Ausschlachtung des Vorsitzes durch ehrgeizige Präsidenten forderte eine breite Koalition, insbesondere kleiner Mitgliedstaaten, die Einführung eines feinmaschigen Netzes konkreter vertraglich garantierter Mechanismen, um unerwartete und potenziell unkontrollierbare Schritte des neuen Präsidenten verhindern zu können. Die Vertragsarchitekten scheinen die Rolle eines effizienten Managers und Konsensstifters als Leitbild verfolgt zu haben, ohne dabei einen dominanten Führungsanspruch innerhalb ihres Klubs und der institutionellen Architektur vertraglich mit harten Befugnissen zu untermauern. Die Rolle des ,ersten Dieners' vieler Herren beschreibt das Amt folglich treffender als das Bild eines neuen ,Hausherrn` über viele Diener oder gar eines vollwertigen Präsidenten (der Europäischen Union).

Im Gegensatz zu manchen Befürchtungen bezüglich des Einflusses von hauptamtlichen Präsidenten auf das allgemeine institutionelle Gleichgewicht der Union aus einer System-

73 Art. 15 Abs. 6 Lit. d EUV.

74 European Council. The President: Speech by the President of the European Council, 2010.

75 Vgl. Klaus Hänsch: Der Konvent - unkonventionell, in: integration 3/2002, S. 226-229.

76 Centre for European Policy Studies/EGMONT/European Policy Centre: The Presidency of the Council, 2007, S. 52. 
perspektive hat der Vertragstext von Lissabon keine hierarchische Vorherrschaft gegenüber dem Rat „Allgemeine Angelegenheiten“ oder dem Kommissionspräsidenten eingeführt und somit einer "schleichenden Intergouvernementalisierung" zumindest dem Buchstaben nach entgegengewirkt. Stärker formuliert platziert der geschriebene Text das Amt in eine prozedurale, inter- und intrainstitutionelle Zwangsjacke, die nur bedingt Spielraum für autonomes oder ehrgeiziges Handeln lässt.

So wird nicht zuletzt der persönliche Stil des Amtsinhabers in der gelebten Tagespolitik künftig von besonderem Interesse für die Beobachter des Präsidenten des Europäischen Rates bleiben. Das Profil des Amtes kann in der gelebten Praxis durch eine starke Persönlichkeit und eine Reihe von informellen Vereinbarungen schrittweise in Richtung einer charismatischen Führungsrolle ausgebaut werden. Der hauptamtliche Präsident könnte hierzu die in der Öffentlichkeit und besonders durch die Presse geweckten Erwartungen an einen „,Mr Europe“" oder „Mrs Europe“ 77 nach innen und außen kultivieren. Bereits in dem gemeinhin vermuteten Wettbewerb um das Amt zwischen dem früheren, international erfahrenen Britischen Premier Tony Blair und dem langjährigen charismatischen ,Europäer" und Luxemburger Premierminister Jean-Claude Juncker sicherte sich der vergleichsweise unbekannte ,Neuling' auf europäischem und internationalem Parkett, Van Rompuy, das Vertrauen der Staats- und Regierungschefs. Hierin mag die Annahme Bestätigung finden, dass eine weniger profilierte und politisch weniger ambitionierte Persönlichkeit das Risiko unbeabsichtigter Folgewirkungen für die Staats- und Regierungschefs minimiert. Es scheint somit in der Natur des Amtes zu liegen, dass eine klar positionierte und möglicherweise polarisierende Führungspersönlichkeit schwieriger die Zustimmung durch die Mitglieder des Europäischen Rates erfahren wird. Die spezifische Erfahrung des Verhandelns und Moderierens im nationalen Kontext des vormaligen belgischen Premiers und neuen Präsidenten ad personam zeichnet ihn somit als effektiven Konsensstifter aus, der dazu den Ruf persönlicher Bescheidenheit genießt.

Exogene Schocks und endogene Krisen können die Nachfrage nach Führungskraft in der Vertragspraxis ebenfalls weiter fördern und den Druck auf den Präsidenten erhöhen, über Absicht und Geist des geschriebenen Vertragstextes sensu stricto hinaus zu handeln. Es kann als eine heimliche Reserve oder Rückfallposition dieses Amtes verstanden werden, Vertragsvorgaben gegebenenfalls flexibler zu interpretieren oder sogar aufzuweichen. In der Vergangenheit haben die Vorsitzenden der Präsidentschaftsstaaten solche unerwarteten Gelegenheiten nicht selten zur Ausbildung ihres Profils genutzt - gegenüber ihren Wählern aber auch in der europäischen und internationalen Arena. Die erbrachten Leistungen in solchen ,Notfällen' konnten die Reputation der jeweiligen Politiker im Kreise ihrer Kollegen aufwerten oder umgekehrt ihre Rolle auf die eines reinen Vorsitzenden reduzieren. Auch eine gestärkte Bereitschaft zur gemeinschaftlichen Problemlösung kann in diesen Fällen gegenüber dem nationalstaatlichen Souveränitätsreflex gestärkt werden und den Präsidenten drängen, eine ausgeprägte Führungsrolle zu übernehmen. Die Mitgliedstaaten als Prinzipale, wie auch die anderen EU-Institutionen, werden diese Entwicklungen jedoch unter Vorbehalt und unter dem Aspekt des Einstimmigkeitserfordernisses genauestens zu prüfen haben.

77 Alain Lamassoure: Histoire secrète de la Convention européenne, Paris 2004, S. 61. 